\title{
The effect of physical training on glutamate transporter expression in an experimental ischemic stroke rat model
}

\author{
Gye-Yeop Kim ${ }^{\text {a }}$, Eun-Jung Kim ${ }^{\mathrm{b}}$ \\ ${ }^{a}$ Department of Physical Therapy, Dongshin University, Naju, Republic of Korea \\ ${ }^{b}$ Department of Physical Therapy, Nambu University, Gwangju, Republic of Korea
}

Objective: The present study was aimed at determining the effect of physical training on glutamate transporter activity in a middle cerebral artery occlusion (MCAO)-induced ischemia injury rat model.

Design: Randomized controlled trial.

Methods: In this study, we randomly divided them into three groups. Group I included non-occlusion sham controls ( $\mathrm{n}=10$ ), Group II included non-physical training after MCAO ( $\mathrm{n}=10)$, and Group III included rats that were subjected to physical training after MCAO $(\mathrm{n}=10)$. Rats in the physical training group underwent treadmill training, which began at $24 \mathrm{~h}$ after MCAO and continued for 14 consecutive days. The training intensity was gradually increased from $5 \mathrm{~m} / \mathrm{min}$ on the first day to $12 \mathrm{~m} / \mathrm{min}$ on day 3 , and it was maintained until day 14. Focal cerebral ischemia was examined in adult male Sprague-Dawley rats by using the MCAO model. We determined the functional outcomes for each rat on days 1, 7, and 14. Glutamate transporter-1 (GLT-1) activity in the cortex of rats from all three groups was examined at the end of the experiment.

Results: Out result show that MCAO rats exhibited severe neurological deficits on the 1 day, and there was no statistically significant in each groups. We observed that the functional outcomes were improved at days 7 and 14 after middle cerebral artery occlusion, and GLT-1 activity was increased in the physical training group $(p<0.05)$.

Conclusions: These results indicated that physical training after focal cerebral ischemia exerts neuroprotective effects against ischemic brain injury by improving motor performance and increasing the levels of GLT-1 activity.

Key Words: Glutamate transporter, Physical training, Stroke

\section{Introduction}

Stroke is a major cause of morbidity and mortality around the world, and there is an association between age and the risk of stroke [1]. Ischemic strokes, accounting for $80 \%$ of all strokes may be caused due to atherosclerotic disease, embolism, hypercoagulable disorders, and in certain cases, due to undetermined causes [2]. It is also one of the leading causes of motor dysfunction, cognitive impairment, movement and gait disorder [3]. The restoration of these main functions is therefore, a major aim in stroke rehabilitation.

Major ischemic brain repair processes during stroke re- covery include neuralplasticity, angiogenesis, and neurogenesis $[4,5]$. These brain repair processes are influenced by environmental stimulation factors such as sensory and motor stimuli through the neuronal pathway. The ischemic brain is the most responsive to these alterations and interacts with the environment to modify its neural circuitry [6]. Physical exercise and training is an effective rehabilitation method for protecting neural cells against ischemia-induced brain injury [7,8]. Physical exercise is known to simultaneously promote the neuronal cell survival mechanisms, while inhibiting the neuronal apoptotic pathways [9]. In addition, it increases capillary density by inducing angiogenic

Received: 9 November, 2013 Revised: 12 December, 2013 Accepted: 15 December, 2013

Corresponding author: Eun-Jung Kim

Department of Physical Therapy, Nambu University, 23 Cheomdanjungang-ro, Gwangsan-gu, Gwangju 506-706, Republic of Korea Tel: 82-61-970-0235 Fax: 82-62-970-0492 E-mail: ddosuny@hanmail.net

(c) This is an Open-Access article distributed under the terms of the Creative Commons Attribution Non-Commercial License (http://creativecommons.org/licens es/by-nc/3.0) which permits unrestricted non-commercial use, distribution, and reproduction in any medium, provided the original work is properly cited.

Copyright $@ 2013$ Korean Academy of Physical Therapy Rehabilitation Science 
growth factors in the brain, and protects against ischemic cell damage [10]. Further, physical activity enhances the neuronal plasticity and the excitability of neuronal pathways. Exercise is involved in the remodeling of neuronal circuits and increased motor function, thus ameliorating pathological conditions $[11,12]$. Considering the several different type of physical training available, it is important to know which of these stroke rehabilitation strategies is the most effective in facilitating motor function recovery [13-15]. Glutamate is the main excitatory neurotransmitter, during the ischemic condition, which mediates a large majority of synaptic transmission and is responsible for the expression of neural plasticity and higher brain functions [16,17]. Glutamate overstimulates N-methyl-D-aspartate receptors, which upon activation mediate calcium influx and acts as the primary factor in calcium-led mechanisms of neuronal cell death and neuroinflammation, following ischemic stroke [18]. The inhibition of glutamate-induced neuronal excitotoxicity has been a therapeutic target for stroke for many years [19]. Several recent studies indicate that physical training also plays an important role in angiogenesis, neuronal plasticity, neuroinflammation, and functional recovery after stroke [20-22].

However, the exact mechanisms underlying the effectiveness of physical training are not yet very clear. Here, we sought to lassess the contribution of physical training in promoting the functional outcome and glutamate transporter activity in focal cerebral ischemic injury rats.

\section{Methods}

\section{Rat middle cerebral artery occlusion rat model}

Thirty male Sprague-Dawley rats (weight $=250-260 \mathrm{~g}$ ) were used in this study. Rats were housed at a temperature of $25.0 \pm 1.0^{\circ} \mathrm{C}$ and $50 \pm 5 \%$ a humidity, with a 12 -h light-dark cycle, and had free access to food and water. The rats were acclimated for one week, and were randomized into three groups. Group I included the non-occlusion sham control rats $(n=10)$, Group II included the rats subjected to non-physical training after middle cerebral artery occlusion $(\mathrm{MCAO})(\mathrm{n}=10)$, and Group III included rats that underwent physical training after MCAO $(n=10)$. All animal experimental protocols were performed in accordance with the guidelines of the institution's animal care and use committee of Dongshin University. Focal cerebral ischemia was induced with a modified intraluminal suture method, which was previously described in detail [23]. Briefly, the left com- mon internal and external carotid arteries were exposed through a midline incision in the neck and then carefully dissected from the surrounding tissues under an operating microscope. After electro-coagulation of the external and common carotid arteries, a 3-0 silicon rubber-coated monofilament was inserted through the common carotid artery into the internal carotid artery to a depth of $18-20 \mathrm{~mm}$ beyond the carotid bifurcation at the base of the middle cerebral artery. An atraumatic aneurysm clip was placed on the internal carotid artery to prevent bleeding. The clip and the monofilament were removed after $1 \mathrm{~h}$ to stimulate transient ischemia, and after $24 \mathrm{~h}$ to stimulate permanent ischemia. Finally, the incision was sutured once the clip and the monofilament were removed. In the Group I, all steps were included except for MCAO procedure.

\section{Treadmill exercise and functional outcomes}

Rats in the physical training group underwent treadmill training $24 \mathrm{~h}$ after MCAO for 14 consecutive days. The training intensity was gradually increased from $5 \mathrm{~m} / \mathrm{min}$ on the first day to $12 \mathrm{~m} / \mathrm{min}$ on day 3 , and it was maintained until day 14, as described in detail in our previous study [24]. On days 1, 7, and 14 after MCAO, neurological deficit scores were tested as previously described, and all rats were scored by an observer blinded to the experiment design with the following criteria: score 0 , no neurological symptoms; score 1, unable to extend right forepaw fully; score 2, reduced grip of the right forelimb; score 3, torso turning to the right side when held by tail; score 4 , circling or walking to the right; score 5, failure to walk without help; score 6, no spontaneous activity or narcosis; and score 7 , dead.

\section{Western blotting analysis}

About $10 \mu \mathrm{g}$ of protein was separated using $12 \%$ sodium dodecylsulfate-polyacrylamide gel electrophoresis after brain tissues were collected, transferred onto a polyvinylidene difluoride membrane, and blocked in 5\% non-fat milk at room temperature for $2 \mathrm{~h}$. Resultant protein was incubated in rabbit anti-GLT-1 (Santa Cruz Biotechnology, Santa Cruz, CA, USA) and $\beta$-actin (Santa Cruz Biotechnology), followed by incubation in horseradish peroxidase-labeled goat anti-rabbit immunoglobin (1:5,000, Zymed, San Francisco, CA, USA) at room temperature for $3 \mathrm{~h}$. Membranes were finally incubated with a chemiluminescent reagent (PerkinElmer Life Sciences, Boston, MA, USA) and the signals produced were recorded on X-ray film (BIOMAX XAR Film, Rochester, NY, USA) for a densitometric analysis. 


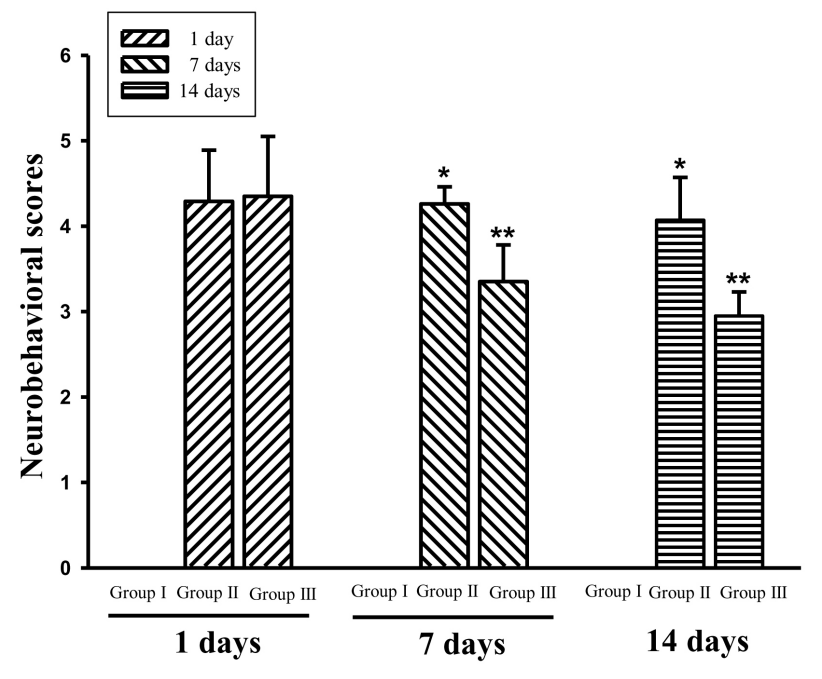

Figure 1. The effect of physical training improved neurobehavioral recovery. Values are presented as mean $(\mathrm{SD})$ of three independent experiments. ${ }^{*} p<0.05$ compared to Group I, ${ }^{* *} p<0.05$ compared to Group II.

\section{Data analysis}

Data analysis was performed with PASW Statistics 18.0 (IBM Co., Armonk, NY, USA). All of the data were expressed as mean \pm standard deviation (SD). Differences in limb placement test scores were examined with a parametric one-way ANOVA and Scheffe's post hoc procedure at 95\% significance levels. For test based on scoring systems, the nonparametric Kruskal-Wallis test was used with the multiple-comparison post hoc test to determine the number and relation of the group differences at a $95 \%$ significance level.

\section{Results}

All rats with MCAO exhibited severe neurological deficits on the first day, and there was no statistically significant difference between the non-physical training group and the physical training group ( $4.29 \pm 0.60$ vs. $4.35 \pm 0.70$ in Group II and Group III, respectively). The effect of physical training on the recovery of function was evaluated at on days 7 (4.26 \pm 0.20 vs. $3.35 \pm 0.43$ in Group II and Group III, respectively) and 14 ( $4.07 \pm 0.50$ vs. $2.95 \pm 0.28$ in Group II and Group III, respectively) after MCAO ( $p<0.05$; Figure 1). We observed that physical training significantly promoted functional outcomes on the 7th and 14th day after MCAO ( $p$ $<0.05$ ). All rats in the Group I evaluated on days 1, 7, and 14 , exhibited no neurological deficits. We analyzed brain fraction extracts from the rats in all three groups at the end of
A

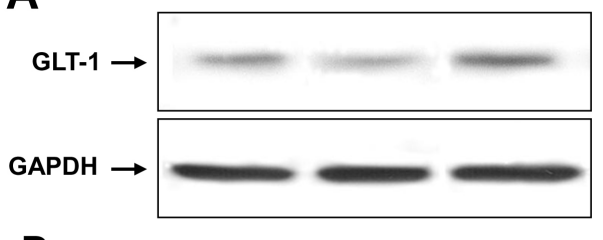

B

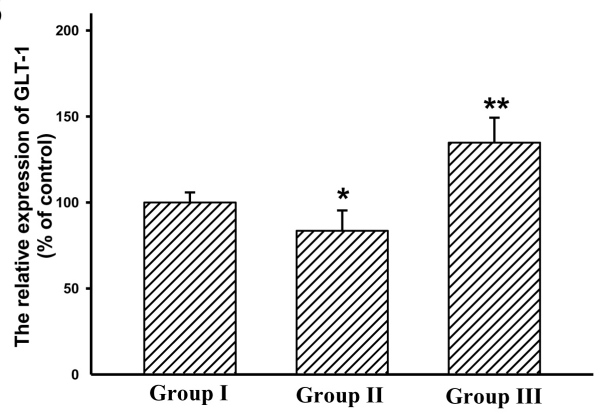

Figure 2. The effect of physical exercise increased expression of glutamate transporter-1 (GLT-1). (A) Representative images of Western blotting for GLT-1 and glyceraldehyde 3-phosphate dehydrogenase (GAPDH). (B) Quantification of the optical density for GLT-1. Values are presented as mean (SD) of three independent experiments. ${ }^{*} p<0.05$ compared to Group I, ${ }^{* *} p<0.05$ compared to Group II.

the experiment to determine glutamate transporter-1 (GLT-1) activity levels. Compared to the Group II, the Group III exhibited an increasing trend in GLT-1 activity. After MCAO, the GLT- 1 activity significantly changed by $83.56 \%$ in the Group II and by $134.84 \%$ in Group III ( $p<0.05$; Figure 2).

\section{Discussion}

About of the strokes is ischemic, and $20 \%$ is hemorrhagic [25]. Patients with stroke experience various symptoms that limit their daily activities, which include physical disability, neglect, memory, cognitive, and sensory impairment [26,27]. Brain ischemia is one of the leading causes for severe disabilities during their lifetime and often leads to irreversible brain damage described by characteristic adenosine triphosphate depletion, neuronal oxidative stress, release of inflammation cytokines and abnormal release of neurotransmitters [28,29].

Physical activity enhanced the promotion of cell survival mechanisms. A number of previous studies have investigated the role of exercise in promoting enhanced cell survival against brain ischemia and improved functional recovery [30,31]. Our result show that physical training significantly promoted the functional outcomes on day 14 after MCAO. The results indicated that exercise improved the re- 
covery of motor function after MCAO. A previous study demonstrated improvement in survival and a marked reduction in brain damage in Sprague-Dawley rats subjected to a two-week moderately intense treadmill activity [32]. However, whether physical training for two weeks could influence the excessive release of glutamate caused by cerebral ischemia is still unknown.

After MCAO, excessive glutamate release could evoke postsynaptic depolarization and cause influx of cation such as calcium and sodium into the cells, thereby inducing neuronal excitotoxicity $[33,34]$. The GLT-1 is a major glutamate transporter that has been shown to exert neuroprotection in various animal models of ischemic injury and motor neuron degeneration [35-37]. In our study, there were significant inhibitory effects on GLT-1 activity observed in the ischemic stroke model, and physical training led to an increased in GLT-1 activity and improvements in motor function. Changes in GLT-1 activity might reflect neuroprotective characteristics that promote the survival of the cerebral cortex, as has been shown in experimental experiments models of focal cerebral ischemia [34]. Improvements in neurological functions indicated that alterations in GLT-1 expression might be correlated with the neuroprotection.

Thus, our data clearly show that physical training appears to act as a major homeostatic regulator of motor function and GLT-1 upregulation. These findings suggest that physical training is important in functional recovery. The present data revealed that physical training promoted functional outcomes and improved the GLT-1 expression in the ischemic region after MCAO, and this study confirms that physical activity is an early physical therapeutic strategy in the rehabilitation of ischemic stroke.

\section{References}

1. Barker-Collo S, Starkey N, Lawes CM, Feigin V, Senior H, Parag V. Neuropsychological profiles of 5-year ischemic stroke survivors by Oxfordshire stroke classification and hemisphere of lesion. Stroke 2012;43:50-5.

2. Ashjazadeh N, Fathi M, Shariat A. Evaluation of homocysteine level as a risk factor among patients with ischemic stroke and its subtypes. Iran J Med Sci 2013;38:233-9.

3. Singh P, Kaur R, Kaur A. Endovascular treatment of acute ischemic stroke. J Neurosci Rural Pract 2013;4:298-303.

4. Yin X, Meng F, Wei W, Li A, Wang Y, Chai Y, et al. Role of mouse nerve growth factor in neural recovery following hypoxic-ischemic brain damage. Int J Clin Exp Med 2013;6:951-5.

5. Font MA, Arboix A, Krupinski J. Angiogenesis, neurogenesis and neuroplasticity in ischemic stroke. Curr Cardiol Rev 2010; 6:238-44.
6. Makara-Studzińska M, Grzywa A, Spila B. Brain plasticity. Pol Merkur Lekarski 2012;32:345-8.

7. Dornbos D 3rd, Ding Y. Mechanisms of neuronal damage and neuroprotection underlying ischemia/reperfusion injury after physical exercise. Curr Drug Targets 2012;13:247-62.

8. Dimyan MA, Cohen LG. Neuroplasticity in the context of motor rehabilitation after stroke. Nat Rev Neurol 2011;7:76-85.

9. Hötting K, Röder B. Beneficial effects of physical exercise on neuroplasticity and cognition. Neurosci Biobehav Rev 2013;37: 2243-57.

10. Ding YH, Luan XD, Li J, Rafols JA, Guthinkonda M, Diaz FG, et al. Exercise-induced overexpression of angiogenic factors and reduction of ischemia/reperfusion injury in stroke. Curr Neurovasc Res 2004;1:411-20.

11. Quaney BM, Boyd LA, McDowd JM, Zahner LH, He J, Mayo MS, et al. Aerobic exercise improves cognition and motor function poststroke. Neurorehabil Neural Repair 2009;23:879-85.

12. Harris JE, Eng JJ. Strength training improves upper-limb function in individuals with stroke: a meta-analysis. Stroke 2010;41: $136-40$

13. Ribeiro T, Britto H, Oliveira D, Silva E, Galvão E, Lindquist A. Effects of treadmill training with partial body weight support and the proprioceptive neuromuscular facilitation method on hemiparetic gait: a randomized controlled study. Eur J Phys Rehabil Med 2013;49:451-61.

14. Stoller O, de Bruin ED, Knols RH, Hunt KJ. Effects of cardiovascular exercise early after stroke: systematic review and meta-analysis. BMC Neurol 2012;12:45.

15. Shaughnessy M, Michael K, Resnick B. Impact of treadmill exercise on efficacy expectations, physical activity, and stroke recovery. J Neurosci Nurs 2012;44:27-35.

16. Foo K, Blumenthal L, Man HY. Regulation of neuronal bioenergy homeostasis by glutamate. Neurochem Int 2012;61:38996.

17. Lee JM, Grabb MC, Zipfel GJ, Choi DW. Brain tissue responses to ischemia. J Clin Invest 2000;106:723-31.

18. Kristián T, Siesjö BK. Calcium in ischemic cell death. Stroke 1998;29:705-18.

19. Garber K. Stroke treatment--light at the end of the tunnel? Nat Biotechnol 2007;25:838-40.

20. Zhang P, Yu H, Zhou N, Zhang J, Wu Y, Zhang Y, et al. Early exercise improves cerebral blood flow through increased angiogenesis in experimental stroke rat model. J Neuroeng Rehabil 2013;10:43.

21. Forrester LW, Wheaton LA, Luft AR. Exercise-mediated locomotor recovery and lower-limb neuroplasticity after stroke. J Rehabil Res Dev 2008;45:205-20.

22. Mattson MP. Glutamate and neurotrophic factors in neuronal plasticity and disease. Ann N Y Acad Sci 2008;1144:97-112.

23. Longa EZ, Weinstein PR, Carlson S, Cummins R. Reversible middle cerebral artery occlusion without craniectomy in rats. Stroke 1989;20:84-91.

24. Zhang P, Zhang Q, Pu H, Wu Y, Bai Y, Vosler PS, et al. Very early-initiated physical rehabilitation protects against ischemic brain injury. Front Biosci (Elite Ed) 2012;4:2476-89.

25. Runchey S, McGee S. Does this patient have a hemorrhagic stroke?: clinical findings distinguishing hemorrhagic stroke from ischemic stroke. JAMA 2010;303:2280-6. 
26. Jehkonen M, Yliranta A, Rasimus S, Saunamäki T. Neglect rehabilitation after stroke. Duodecim 2013;129:506-13.

27. Li W, Huang R, Shetty RA, Thangthaeng N, Liu R, Chen Z, et al. Transient focal cerebral ischemia induces long-term cognitive function deficit in an experimental ischemic stroke model. Neurobiol Dis 2013;59:18-25.

28. Sánchez-Mendoza E, Bellver-Landete V, González MP, Merino JJ, Martínez-Murillo R, Oset-Gasque MJ. Brain repair after ischemic stroke: role of neurotransmitters in post-ischemic neurogenesis. Rev Neurol 2012;55:533-42.

29. Namura S, Ooboshi H, Liu J, Yenari MA. Neuroprotection after cerebral ischemia. Ann N Y Acad Sci 2013;1278:25-32.

30. Arrick DM, Sun H, Mayhan WG. Influence of exercise training on ischemic brain injury in type 1 diabetic rats. J Appl Physiol (1985) 2012;113:1121-7.

31. Enzinger C, Dawes H, Johansen-Berg H, Wade D, Bogdanovic $\mathrm{M}$, Collett J, et al. Brain activity changes associated with treadmill training after stroke. Stroke 2009;40:2460-7.

32. Cechetti F, Rhod A, Simão F, Santin K, Salbego C, Netto CA, et al. Effect of treadmill exercise on cell damage in rat hippocampal slices submitted to oxygen and glucose deprivation. Brain Res 2007; 1157:121-5.

33. Smith WS. Pathophysiology of focal cerebral ischemia: a therapeutic perspective. J Vasc Interv Radiol 2004;15:S3-12.

34. Shen H, Chen GJ, Harvey BK, Bickford PC, Wang Y. Inosine reduces ischemic brain injury in rats. Stroke 2005;36:654-9.

35. Ketheeswaranathan P, Turner NA, Spary EJ, Batten TF, McColl $\mathrm{BW}$, Saha $\mathrm{S}$. Changes in glutamate transporter expression in mouse forebrain areas following focal ischemia. Brain Res 2011; 1418:93-103.

36. Namura S, Maeno H, Takami S, Jiang XF, Kamichi S, Wada K, et al. Inhibition of glial glutamate transporter GLT-1 augments brain edema after transient focal cerebral ischemia in mice. Neurosci Lett 2002;324:117-20.

37. Verma R, Mishra V, Sasmal D, Raghubir R. Pharmacological evaluation of glutamate transporter 1 (GLT-1) mediated neuroprotection following cerebral ischemia/reperfusion injury. Eur J Pharmacol 2010;638:65-71. 